\title{
Efficacy of standard treatment protocol in recently diagnosed Lupus Nephritis at our tertiary care teaching hospital
}

\author{
Dhakate T. ${ }^{1}{ }^{*}$, Rajadhyaksha A. ${ }^{2}$, Panjwani R. ${ }^{3}$ \\ DOI: https://doi.org/10.17511/ijmrr.2021.i03.01 \\ 1* Tushar Dhakate, Assistant Professor, Department of Nephrology, SSH, NSCB Medical College, Jabalpur, Madhya Pradesh, India. \\ 2 Anjali Rajadhyaksha, Professor, Department of Medicine, Seth G.S. Medical College, Mumbai, Maharastra, India. \\ 3 Ramiz Panjwani, Assistant Professor, Department of Nephrology, VBCH and Namo Medical college, Silvassa, Dadra and Nagar Haveli and \\ Daman and Diu, India.
}

Introduction: Lupus Nephritis occurred in approximately 50\% of Systemic Lupus Erythematosus patients at some point during their illness and is associated with a poor prognosis. Material and Method: A prospective observational study of 50 newly diagnosed LN cases was conducted to investigate the response of standard treatment protocol (Cyclophosphamide -NIH protocol and Mycophenolate Mofetil-MMF). Results: Of the 50 newly diagnosed cases of LN, $94 \%(n=47)$ were females, and $6 \%(n=3)$ were males, with class IV LN accounting for the majority of patients 69.39 $\%(n=34)$. At six months, $36.7 \%(n=11)$ of patients in the cyclophosphamide (CYP) group had a complete response. Only $27.3 \%$ of patients in the MMF group had a complete response; however, this difference was not statistically significant. At the end of one year, only $56.7 \%$ of the CYP group and $81.8 \%$ of the MMF group had a complete response; however, this difference was not statistically significant $(p=0.282)$. Although the initial response with CYP was better and later in the MMF group, these differences were not significant statistically. Tuberculosis or its reactivation was the most common complication during treatment, either with MMF or CYP. One patient died due to latent tuberculosis reactivation, another as a result of severe disease activity at presentation (proteinuria was $20 \mathrm{gm} / 24$ hours in that patient), and the third as a result of pneumonia with septicemia. Conclusion: Treatment with either CYP or MMF is equally effective, but underlying infection, particularly tuberculosis, should be ruled out before initiating therapy.

Keywords: Lupus Nephritis, Systemic lupus erythematosus, Cyclophosphamide, Mycophenolate mofetil

Corresponding Author

Tushar Dhakate, Assistant Professor, Department of Nephrology, SSH, NSCB Medical College, Jabalpur, Madhya Pradesh, India.

Email: tushardhakate86@gmail.com

\section{How to Cite this Article}

Dhakate T, Rajadhyaksha A, Panjwani R. Efficacy of standard treatment protocol in recently diagnosed Lupus Nephritis at our tertiary care teaching hospital. Int J Med Res Rev. 2021;9(3):135-140. Available From https://ijmrr.medresearch.in/index.php/ijmrr/article/ view/1283
To Browse

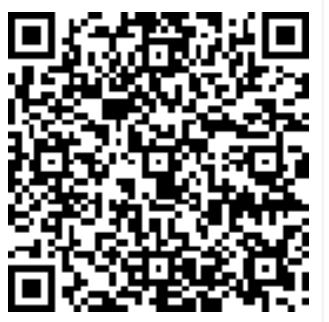

Manuscript Received 2021-04-25

Conflict of Interest No
Review Round 1 2021-05-05

Funding Nil

Review Round 2
2021-05-09
Ethical Approval
Yes

Review Round 2 Yes
Review Round 3

Plagiarism X-checker $9 \%$
Accepted 2021-05-18

Note

(C) 2021 by Tushar Dhakate, Anjali Rajadhyaksha, Ramiz Panjwani and Published by Siddharth Health Research and Social Welfare Society. This is an Open Access article licensed under a Creative Commons Attribution 4.0 International License ttps://creativecommons.org/licenses/by/4.0/ unported [CC BY 4.0]. 


\section{Introduction}

Renal glomeruli are the most involved structure, presenting as lupus nephritis (LN) in Systemic Lupus Erythematosus (SLE). Immune complex formation and deposition - circulating or in situ - in the mesangium, subepithelial, or subendothelial layers initiate the pathogenic events that result in histopathological changes in the glomeruli. The renal disease occurs in up to $25-60 \%$ of SLE patients, most frequently within five years of disease onset.

It is regarded as one of the most powerful predictors of an unfavourable outcome, which eventually affects about half of all patients at some point during their illness.[1,2]. LN defined as per SLEDAI definition $[3,4]$. if any of the four following criteria are met; 1 . Urinary casts (Heme-granular or red blood cells casts.) 2. Haematuria ( $>5$ red blood cells/high power field, excluding stone, infection, and other causes). 3. Proteinuria ( $>0.5 \mathrm{~g} / 24 \mathrm{~h}$, regarding new-onset or recent increase of $>0.5 \mathrm{~g} / 24 \mathrm{~h}) .4$.

Pyuria ( $>5$ white blood cells/high power field, excluding infection). LN classified into six classes by the International Society of Nephrology and Renal Pathology Society (ISN/RPS) to provide a more concise description of various lesions and classes of LN.[5]. We conducted a Prospective observational study to investigate the response of standard treatment protocol (CYP -NIH protocol and MMF)

\section{Aims And Objectives}

To study the response of $L N$ to standard treatment at the end of the study

- response in clinical and laboratory parameter over one year follow up

- assess the frequency of total and partial remission at six months and 12 months

\section{Material and Methods}

Study design: An observational and prospective study from Aug 2010 to Aug 2012. The study was approved by the Institutional Ethics Committee.

Setting - From August 2010 to August 2012, fifty newly diagnosed LN cases were included in the study. All of these LN patients met the ACR's 1997 revised SLE classification criteria.[6]. For one year, all patients were followed up at least three times a month, and more frequently if necessary.

\section{Inclusion criteria:}

1) Patients diagnosed as SLE based on the American College of Rheumatology (ACR) criteria.

2) Patients (or their guardians) should give informed consent for the investigations.

3) Age $>12$ yrs.

Exclusion criteria: Patients with 1) Already diagnosed with Lupus Nephritis 2) Overlapping feature of other Glomerulonephritis 3) Pregnancy 4) HIV infection

Consent: All patients or their relatives/guardians provided written informed consent for blood sampling and renal biopsy. Study procedure: In this study, 50 consecutive subjects who met the inclusion criteria were recruited over a year and then followed up for another year.

History: During the follow-up, the onset, duration, and progression of all symptoms and clinical manifestations were recorded. Clinical examination: At each visit, general and systemic examinations were performed. Investigation and follow up: Routine investigations: Complete hemogram, Erythrocyte sedimentation rate(ESR), Renal function tests, Liver function tests, serum uric acid, Total protein, albumin, Fasting, and postprandial blood sugar, Lipid profile, HIV, CPK(Creatine phosphokinase) whenever indicated, Thyroid function test, Urine routine, and microscopic examination, 24-hour urinary protein, Chest radiograph- posteroanterior view, ECG,

Special Investigations: ANA by immunofluorescence Anti ds-DNA by indirect immunofluorescence C3 and C4 complement levels (with BNProSpec KIT by nephelometric assay with normal range of $\mathrm{C} 3$ 90- $180 \mathrm{mg} / \mathrm{dl}$ and C4- $15-45 \mathrm{mg} / \mathrm{dl}$ ) ACLA (anticardiolipin antibody) IgG and IgM ANA blot if necessary ANCA (Antineutrophil cytoplasmic antibody) Direct and indirect Coomb"s test if required. USG (Ultrasonography) guided renal biopsy - All newly diagnosed cases of lupus nephritis are subjected to USG guided renal biopsy and are classified into six groups based on the ISN/RPS 2003 classification of LN. Histological criteria were used to determine activity (maximum score, 24 points) and chronicity indices (maximum score, 12 points). Other tests, such as 2D ECHO, USG abdomen and pelvis, computed tomography (CT), magnetic resonance imaging (MRI), and cerebrospinal fluid examination, were performed as needed. 
Clinical evaluations and laboratory tests were performed on all patients at the start and threemonth intervals, or more frequently if necessary. In addition, they were evaluated and managed for any complaints or complications that arose between these visits. All patients were followed from the time they were diagnosed with lupus nephritis until one year later.

Management: The patients were investigated and treated according to the standard LN treatment regimen. There were no experimental treatments, and the patient did not face any potential financial hardship due to this research. All patients received treatment as per standard protocol. All patients received Tab Prednisolone, Tab Aspirin, Tab. Hydroxychloroquine, statins, Vitamin D, Calcium supplements, ACE inhibitors, or blockers. Either Pulse Cyclophosphamide (CYP) therapy (as per NIH protocol) or Tab MMF (as per ALMS trial ) was used for induction (for class III, IV, and V) after counselling about the advantage and disadvantages of each of these.[7,8]. Analysis: All data were entered into an Excel spreadsheet, and a descriptive analysis of the demographic and clinical profiles was performed using Mean, Range, and Cumulative Frequency as a percentage. The repeated test ANOVA was used to compare groups, and a $p$-value of 0.05 was considered statistically significant.

\section{Results}

Of the 50 newly diagnosed cases of LN, 94\% ( $n=47)$ were females, and $6 \%(n=3)$ were males with a female to male sex ratio was $15.67: 1$. The maximum number of patients were in between the 20-29 age groups. Out of 50 newly diagnosed cases of LN, most patients had class IV LN 69.39\% $(n=34)$ followed by Class II LN $14.29 \%(n=7)$, Class III and V LN $6.12 \%$ each $(n=3)$. Class VI and class I LN each $2.04 \%(n=1)$, renal biopsy was not performed in one female patient because was expired soon after admission because of severe disease activity.

All 50 patients were ANA positive, out of which the most common pattern being homogeneous (60\%) followed by Speckled (26\%), Nucleolar (6 \%), Cytoplasmic (4\%), and Centromere (4\%) All patients received treatment as per standard protocol as mentioned above. Out of 50 patients enrolled,64\% $(n=32)$ were on Cyclophosphamide pulse regimen (NIH Regimen), 22\% $(\mathrm{N}=11)$ were on MMF, $12 \%(n=6)$ were only on Tab Prednisolone
(Class I and II LN), and one patient was on maintenance hemodialysis as diagnosed to have ESRD. The response to induction therapy either with Cyclophosphamide or MMF was assessed statistically in terms of complete remission, partial remission, and no response. Complete remission defined as urinary protein $<500 \mathrm{mg} /$ day with normal urinary RBC, normal serum albumin 3.5-5.5 gm/dl, and normal serum Creatinine.

Partial remission defined as stabilization or improvement of serum creatinine, RBC in urine $<5$ cells/field, and persistent 24-hour reduction (if nephrotic, reduction more than or equal to $50 \%$ but with a value lower than $3 \mathrm{gm} / 24$ hours; if nonnephrotic, reduction more than or equal to $50 \%$; but with a value greater than $500 \mathrm{mg} / 24$ hours). At six months of treatment, complete response was seen in $36.7 \%(n=11)$ in the cyclophosphamide group and only in $27.3 \%$ of patients in the MMF group, but this difference was not statistically significant. $(P=0.614)$. At the end of one year, the complete response was seen in only $56.7 \%$ in the cyclophosphamide group and $81.8 \%$ in the MMF group; however, this difference was also not statistically significant $(p=0.282)$ Though initial response was better with cyclophosphamide and later in MMF group, these were not statistically. significant. In the Cyclophosphamide group, out of 32,2 patients died before six months, so excluded from the analysis of response to treatment.

Table 1-Partial and Complete response to CYP and MMF at 6 months and 12 months

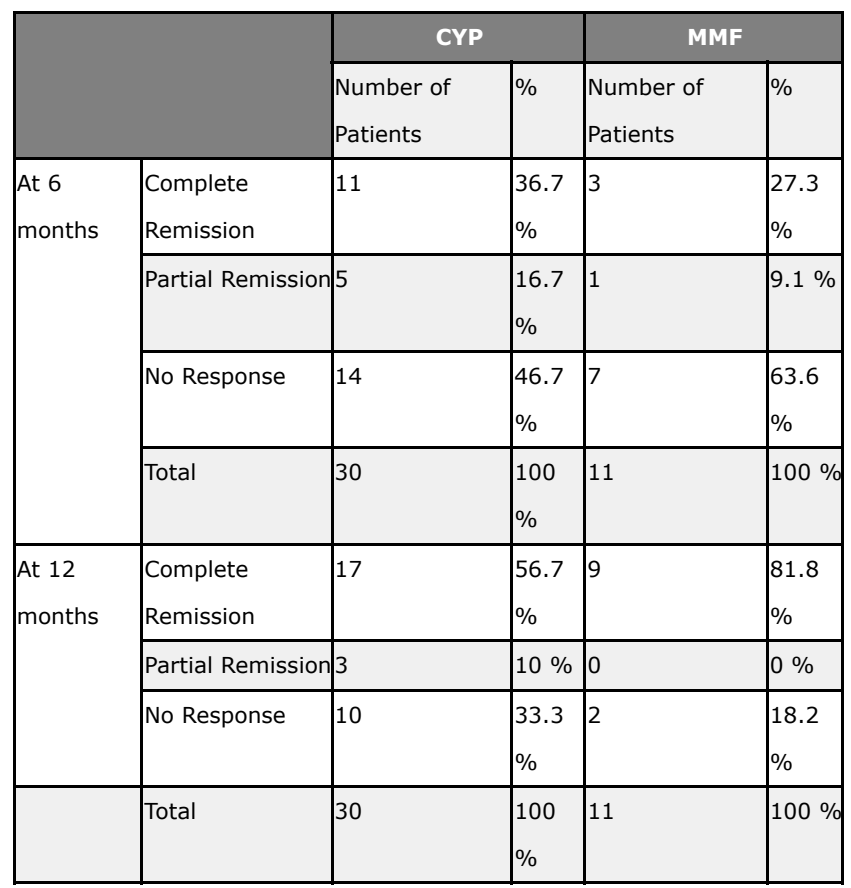


The mean of 24 hours urinary protein at the enrollment of study was $2607.61 \mathrm{gm} / 24$ hours with a maximum being $20000 \mathrm{gm} / 24$ hours and minimum being $435 \mathrm{gm} / 24$ hours and at the end of study mean was $492.25 \mathrm{gm} / 24$ hours with a maximum of $2428 \mathrm{gm} / 24$ hours and minimum being $97 \mathrm{gm} / 24$ hours. The nephrotic range proteinuria was present in $20 \%(n=10)$ of patients, $70 \%$ of which belonged to class IV LN $(n=7)$, one patient had class V LN, one had VI LN, one died because of severe disease activity which had proteinuria 20 $\mathrm{gm} / 24 \mathrm{hrs}$.

After excluding three which were expired during follow-up, there was a significant decrease in 24 hours urinary protein after treatment ( $p<0.001)$. The repeated measure ANOVA was used for analysis. The C3 and C4 significantly increase the following treatment after the end of one year from a mean of 48.91 and 12.856 respectively to a mean of 130.13 and 36.45. (p-value 0.001 by using repeated ANOVA measure test.

All 50 patients were ANA positive with the different patterns as mentioned above, out of which 54\% $(n=27$ were ds DNA positive) after the end of 1-year ds DNA positivity decreased to $14.89 \%(n=7)$ with treatment which was statistically significant. Out of 50 patient, $24 \%$ patients $(n=12)$ developed complication during one year period, which was as follows

Table 2- Complication occurred during followup of LN patients over one year.

\begin{tabular}{|l|l|}
\hline \multicolumn{1}{|c|}{ Complication } & \multicolumn{1}{c|}{ Number of patients } \\
\hline Pulmonary tuberculosis & 2 \\
\hline Abdominal Koch's & 1 \\
\hline Reactivation of tuberculosis & 2 \\
\hline TB Lymphadenitis & 1 \\
\hline Pancreatitis & 2 \\
\hline Avascular Necrosis of the hip joint & 1 \\
\hline Chickenpox infection & 1 \\
\hline HZV infection & 1 \\
\hline Thigh abscess & 1 \\
\hline Total & 12 \\
\hline
\end{tabular}

At the end of one year: Living: $94 \%(n=47)$ Death: $6 \%(n=3)$ All death occurs within six months of diagnosis of LN. Out of 3 patients who expired, one died because of reactivation of latent TB, one had died because of severe disease activity at presentation, proteinuria was $20 \mathrm{gm} / 24$ hours in that patient, and the remaining one died because of pneumonia with septicemia.

\section{Discussion}

In our study, a total of 50 newly diagnosed cases of LN patients based on ACR criteria were included. These were followed up prospectively quarterly for 1 year, more frequently if necessary. In our study, 94 $\% \quad(n=47)$ of newly diagnosed $L N$ cases were female, while $6 \%(n=3)$ were male. The female to male ratio was $15.67: 1$. The average age at onset of LN was $26.18 \pm 8.39$ years. The majority of patients (54\%) $(n=27)$ were between the ages of 20 and 29, with only $8 \%(n=4)$ over the age of 40 . The $M: F$ ratio indicated a female predominance, but different ratios in different study groups could be due to differences in geographic area, genetic and environmental factors. The demographic profile at the time of LN diagnosis was comparable to other studies. $[1,7]$.

The most common histological class was class IV $(69.39 \%)$, similar to the other study, including those in India.[1,8,9]. Renal biopsy was not done in one patient as she expired soon after diagnosis of SLE because of severe disease activity. The mean of 24 Hours urinary protein at the start of the study was $2607.61 \mathrm{gm} / 24$ hours, which reduced to 492.25 $\mathrm{gm} / 24$ hours at the end. Proteinuria in the nephrotic range was present in $20 \%(n=10)$ of patients, $70 \%$ $(n=7)$ belonged to class IV LN, one patient had class V LN, one had VI LN, and one died due to severe disease activity with proteinuria of $20 \mathrm{gm} / 24$ hrs. In the study by C Chrysochou et al. [10], $33 \%$ had nephrotic range proteinuria, with $27 \%$ having class IV LN, $64 \%$ having class V LN, and \% having class III LN.

According to studies, Indians have a higher percentage of nephrotic range proteinuria. Dhir et al [1]. found that $34.4 \%$ in their study (north India) had nephrotic range proteinuria. In Raphael V's (Northeast India) study, $66.6 \%$ had nephrotic range proteinuria. Both of the above Indian studies were retrospective. In our study, the percentage of nephrotic range proteinuria was only $20 \%$ which could be because our study was prospective, which helps to detect proteinuria earlier and early initiation of appropriate treatments. We analyzed the treatment response in class III, IV, V LN who were either on cyclophosphamide or MMF for induction at six months and 12 months. (one patient with class II also included as was on cyclophosphamide for concomitant ILD), with the exclusion of those patients who died during the follow-up period. 
At six months of treatment, complete response was seen in $36.7 \%(n=11)$ in the cyclophosphamide group and only in $27.3 \%$ of patients in the MMF group, but this difference was not statistically significant. $(P=0.614)$. At the end of one year, the complete response was seen in only $56.7 \%$ in the cyclophosphamide group and $81.8 \%$ in the MMF group; however, this difference was also not statistically significant $(p=0.282)$ Though initial response was better with cyclophosphamide and later in MMF group, these were not statistically significant. In the study by Dhir et al.[1]. at the end of one year, out of 130 patients, 71 patients $(54.62 \%)$, partial remission was seen in 39 patients (30\%).In our study, overall, at six months, the complete response rate was $34.78 \%$ (16 out of 46 including those on prednisolone only), and the partial response rate was $52.17 \%$.

At the end of our study, i.e., after one year, the complete response was seen in $60.87 \%$ (28 out of 46 patients including those on prednisolone only), and partial response seen in $30.43 \%$ (14 out of 46 patients). The result of our study is similar to that seen in Dhir et al. group with a slightly higher response rate may because our study is prospective and only 50 patients studied as compared with the above study, which is retrospective and contained relatively large number of patients. The most common complication being tuberculosis or reactivation of latent $\mathrm{TB}$. Therefore each patient should be screened for underlying tuberculosis before starting an immunosuppressant. Also, pancreatitis was quite common.

Therefore abdominal pain in the case of SLE should be investigated for pancreatitis. The complication has been seen most commonly in the patient treated with cyclophosphamide $83.33 \% \quad(n=12)$ than that of MMF $16.67 \%(n=2)$. Therefore one should be considered the risk-benefit ratio and patients affordability before initiating inductions. Among the 3 patients who died, the causes were: reactivation of underlying tuberculosis in one patient (had class III LN), pneumonia with septicemia in one patient (had class IV LN), one death of severe disease activity at presentation (biopsy was not done). In the study by Dhir et al.[1].

Among the 16 patients who died, the causes were infections in 8 (sepsis in 4 , disseminated tuberculosis in 3, and pneumonia in 1), subdural hematoma in 1 (on anticoagulation), severe bone marrow aplasia with pulmonary hemorrhage in 1 , acute abdomen in 1 (unknown cause), post-surgery
Sudden death in 1 , diabetic ketoacidosis in 1 , suicide in 1 , and unknown in 2 ( 1 of whom was in renal failure). In the study by Cevera $\mathrm{R}$ et al. "Morbidity and mortality in systemic lupus erythematosus during 10 years: a comparison of early and late manifestations in a cohort of 1,000 patients", the most frequent causes of death were active SLE(26.5\%), thrombosis(26.5\%) and infections(25\%) with active SLE and infections appeared to be a most common cause of death during initial 5 years period while thrombosis became a most common cause of death during last 5 years.[2]. As a developing country, the main cause of death being infection which correlated with other Indian studies.

\section{Conclusion}

Treatment with either CYP or MMF is equally effective, but underlying infection, particularly tuberculosis, should be ruled out before starting therapy.

\section{What does the study add to existing knowledge?}

Therapy with either CYP or MMF is equally effective in the treatment of Lupus.

\section{Author contributions}

$T D, A R$ collected the data and conducted this study. RP and TD did data analysis. TD, AR and RP did manuscript drafting. All authors were involved in revising and approved the final version of the manuscript.

\section{Reference}

01. Dhir V, Aggarwal A, Lawrence A, Agarwal V, Misra R. Long-term outcome of lupus nephritis in Asian Indians. Arthritis Care Res (Hoboken). 2012 May;64(5)713-20. doi: 10.1002/acr.21597 [Crossref][PubMed][Google Scholar]

02. Cervera R, Khamashta MA, Font J, Sebastiani GD, Gil A, Lavilla P, et al. Morbidity and mortality in systemic lupus erythematosus during a 10-year period- a comparison of early and late manifestations in a cohort of 1,000 patients. Medicine (Baltimore). 2003 Sep;82(5)299-308. doi: 10.1097/01.md.0000091181.93122.55 [Crossref] [PubMed][Google Scholar] 
03. Gladman D, Ginzler E, Goldsmith C, Fortin P, Liang $M$, Urowitz $M$, Bacon $P$, Bombardieri S, Hanly J, Hay $E$, et al. Systemic lupus international collaborative clinics- development of a damage index in systemic lupus erythematosus. J Rheumatol. 1992 Nov;19(11)1820-1. [Crossref] [PubMed][Google Scholar]

04. Gladman D, Ginzler E, Goldsmith C, Fortin P, Liang $M$, Urowitz $M$, et al. The development and initial validation of the Systemic Lupus International Collaborating Clinics/American College of Rheumatology damage index for systemic lupus erythematosus. Arthritis Rheum. 1996 Mar;39(3)363-9. doi: 10.1002/art.1780390303 [Crossref][PubMed][Google Scholar]

05. Weening JJ, D'Agati VD, Schwartz MM, Seshan SV, Alpers CE, Appel GB, et al. The classification of glomerulonephritis in systemic lupus erythematosus revisited. J Am Soc Nephrol. 2004 Feb;15(2)241-50. doi: $\quad$ 10.1097/01.asn.0000108969.21691.5d [Crossref][PubMed][Google Scholar]

06. Hochberg MC. Updating the American College of Rheumatology revised criteria for the classification of systemic lupus erythematosus. Arthritis Rheum. 1997 Sep;40(9)1725. doi: 10.1002/art.1780400928 [Crossref][PubMed][Google Scholar]

07. Appel GB, Contreras G, Dooley MA, Ginzler EM, Isenberg $D$, Jayne $D$, et al. Mycophenolate mofetil versus cyclophosphamide for induction treatment of lupus nephritis. J Am Soc Nephrol. 2009 May;20(5)1103-12. doi: 10.1681/ASN.2008101028 [Crossref][PubMed][Google Scholar]
08. Austin HA 3rd, Klippel JH, Balow JE, le Riche NG, Steinberg AD, Plotz PH, Decker JL. Therapy of lupus nephritis. Controlled trial of prednisone and cytotoxic drugs. N Engl J Med. 1986 Mar 6;314(10)614-9. doi: 10.1056/NEJM198603063141004 [Crossref] [PubMed][Google Scholar]

09. Hsu CY, Chiu WC, Yang TS, Chen CJ, Chen YC, Lai HM, et al. Age- and gender-related long-term renal outcome in patients with lupus nephritis. Lupus. 2011 Oct;20(11)1135-41. doi: 10.1177/0961203311404912 [Crossref][PubMed] [Google Scholar]

10. Faurschou $M$, Dreyer $L$, Kamper $A L$, Starklint $H$, Jacobsen S. Long-term mortality and renal outcome in a cohort of 100 patients with lupus nephritis. Arthritis Care Res (Hoboken). 2010 Jun;62(6)87380. doi: 10.1002/acr.20116 [Crossref][PubMed] [Google Scholar]

11. Sharma M, Das HJ, Doley PK, Mahanta PJ. Clinical and histopathological profile of lupus nephritis and response to treatment with cyclophosphamide- A single center study. Saudi J Kidney Dis Transpl. 2019 Mar-Apr;30(2)501-507. doi: 10.4103/1319-2442.256857 [Crossref] [PubMed][Google Scholar] 